The Astrophysical JouRnal, 505:159-173, 1998 September 20

(C) 1998. The American Astronomical Society. All rights reserved. Printed in U.S.A.

\title{
INCLINED GAS DISKS IN THE LENTICULAR SEYFERT GALAXY NGC $5252^{1}$
}

\author{
JON A. MORSE ${ }^{2}$
}

Center for Astrophysics and Space Astronomy, University of Colorado, Campus Box 389, Boulder, CO 80309; morsey@casa.colorado.edu

Gerald CECIL

Department of Physics and Astronomy, University of North Carolina, Phillips Hall, CB 3255, Chapel Hill, NC 27599-3255; cecil@wrath.physics.unc.edu

ANDREW S. WILSON ${ }^{2,3}$

Astronomy Department, University of Maryland, College Park, MD 20742; wilson@astro.umd.edu

\section{AND}

Zlatan I. TSVETANOV

Johns Hopkins University, Department of Physics and Astronomy, Center for Astrophysical Sciences, Baltimore, MD 21218; ztsvetanov@pha.jhu.edu Received 1997 December 18; accepted 1998 April 28

\begin{abstract}
We discuss the morphology and kinematics of the extended gas in the type 2 Seyfert galaxy NGC 5252 based on Hubble Space Telescope (HST) WFPC2 continuum and emission-line images (including a new [O III] 25007 image) and a ground-based Fabry-Perot (F-P) velocity map of the ionized gas. The fine-scale morphology of the ionized gas in this galaxy's very extended $(\sim 40 \mathrm{kpc})$ ionization bicone consists of a complex network of filamentary strands. The new WFPC2 [O III] image also reveals more detail in the circumnuclear $(\sim 3 \mathrm{kpc})$ gas disk than is seen in the $\mathrm{H} \alpha+[\mathrm{N}$ II] image presented previously by Tsvetanov and coworkers. The F-P velocity map shows an obvious antisymmetry of the velocity field of the ionized gas across the nucleus. We conclude that there are three dynamical components to the extended gas in NGC 5252. Two of these components are gas disks aligned with the stellar disk, one rotating with the stars and the other counterrotating. The third component is the circumnuclear gas disk seen in the HST observations and its extension to larger scales; this disk has an inclination of $\sim 40^{\circ}$ and a kinematic major axis in P.A. $\sim 90^{\circ}-135^{\circ}$, some $80^{\circ}-125^{\circ}$ from the major axis of the stellar disk. This simple model of two inclined rotating disks, superposed along the line of sight, describes well the seemingly complex kinematics observed in the optical emission lines and the $\mathrm{H}$ I $21 \mathrm{~cm}$ radio maps. The large misalignment between the second disk and the stellar disk suggests that the gas distribution, and possibly the nuclear activity, in NGC 5252 may have resulted from a galaxy merger event. The absence of significant radial motions, together with the well-defined ionization cones, strongly suggests that the gas is photoionized by a compact nuclear source rather than being ionized in situ by shock waves in a largescale outflow.
\end{abstract}

Subject headings: galaxies: elliptical and lenticular, $\mathrm{cD}$ - galaxies: kinematics and dynamics galaxies: Seyfert - galaxies: structure

\section{INTRODUCTION}

The ionized gas comprising the extended emission-line regions of active galaxies is generally presumed to represent ambient interstellar gas excited by the active nucleus. In late-type galaxies, the interstellar medium is intrinsic to the galaxy disk, while in early-type galaxies (E and S0) it may have an external origin, such as a merger with a gas-rich galaxy. An active galactic nucleus (AGN) ionizes and excites this gas; the resulting emission lines can be studied with high spatial resolution at optical and UV wavelengths, providing a wealth of information on the physical properties, chemical abundances, kinematics, and the nature of the excitation. Close to the AGN, the kinematics are affected by the nuclear activity, but farther away gravitational forces should dominate the motions. Thus, studies of large, extended emission-line regions can probe the settling of gas acquired by an early-type galaxy in a merger and the rele-

\footnotetext{
${ }^{1}$ Based in part on observations with the NASA ESA Hubble Space Telescope, obtained at the Space Telescope Science Institute, which is operated by AURA, Inc., under NASA contract NAS 5-26555.

2 Visiting Astronomer, Cerro Tololo Inter-American Observatory, which is operated by AURA, Inc., under contract to the National Science Foundation.

${ }^{3}$ Adjunct Astronomer, Space Telescope Science Institute, 3700 San Martin Drive, Baltimore, MD 21218.
}

vance of such mergers for initiating nuclear activity. Furthermore, the line ratios and kinematics provide keys to the issue of whether the line emission is excited through photoionization by the active nucleus (e.g., Ho, Filippenko, \& Sargent 1993; Binette, Wilson, \& Storchi-Bergmann 1996) or through high-velocity shocks generated at the interface between an outflow (e.g., a radio jet) and the interstellar medium (e.g., Dopita \& Sutherland 1995). In the former case, the properties of the nebulosity can indicate any anisotropy in the ionizing radiation escaping from the nucleus.

In this paper, we present a detailed investigation of the morphology and kinematics of the extended emission-line region of the early-type (S0) Seyfert 2 galaxy NGC 5252. The large angular extent of the ionized gas, distributed in a remarkable bicone extending $\gtrsim 50^{\prime \prime}$ ( $>20 \mathrm{kpc}$, assuming $H_{0}=75 \mathrm{~km} \mathrm{~s}^{-1} \mathrm{Mpc}^{-1}$ ) from the nucleus (Tadhunter \& Tsvetanov 1989; hereafter TT89), arguably provides a unique opportunity for investigating the above issues. Acosta-Pulido et al. (1996; hereafter A-P96) have presented long-slit spectroscopy of the nebulosity, obtaining physical parameters of the gas, including evidence for matterbounded clouds, and properties of the ionizing radiation. The $21 \mathrm{~cm} \mathrm{H}$ I distribution in NGC 5252 has been mapped by Prieto \& Freudling $(1993,1996)$, who found that the 
neutral gas avoids the ionization bicone, suggesting that the large-scale biconical structure is ionization bounded. Wilson \& Tsvetanov (1994; hereafter WT94) mapped a 2" $(900 \mathrm{pc})$ linear radio source that aligns with the bicone axis. They also found a point radio source with a relatively flat spectrum $\sim 22$ " north of the nucleus. Tsvetanov et al. (1996; hereafter TMWC) have presented Hubble Space Telescope (HST) images revealing an ionized, spiral gas disk of diameter $\sim 7^{\prime \prime}(\sim 3 \mathrm{kpc})$ surrounding three colinear clumps of ionized gas within the central $1^{\prime \prime}(450 \mathrm{pc})$ of the galaxy. The spiral arms on the northwest side of the gas disk are seen in absorption against the background of the stellar disk, while those on the southeast side are not, suggesting that the spiral gas disk is substantially inclined with respect to the stellar disk.

Here we continue our study of the extended gas in NGC 5252. We have mapped the two-dimensional velocity field of the ionized gas with an imaging Fabry-Perot (F-P) spectrometer. F-P spectrometers are efficient tools for investigating the kinematics of spatially extended line emission with seeing-limited angular resolution (e.g., Cecil, Bland, \& Tully 1990; Hartigan et al. 1993; Morse, Winkler, \& Kirshner 1995). We have also obtained a new [O III] $\lambda 5007$ image of NGC 5252 with $H S T$, which we combine with our previous HST images to characterize the fine-scale morphology of the ionized gas.

Combining the two-dimensional F-P velocity field with the high-resolution HST images and $21 \mathrm{~cm} \mathrm{H}$ I data of Prieto \& Freudling, we address the following questions: (1) What is the relationship, if any, between the kiloparsecscale spiral gas disk and the tens of kiloparsecs-scale ionized arcs within the bicone? (2) Are the velocity field and emission-line widths consistent with gravitationallydominated motions, or do they reflect outflow from the galaxy nucleus? (3) Do the kinematics suggest a common origin for the ionized arcs and the neutral gas observed in $21 \mathrm{~cm}$ emission?

Throughout this paper, we assume a distance to NGC 5252 of $92 \mathrm{Mpc}\left(H_{0}=75 \mathrm{~km} \mathrm{~s}^{-1} \mathrm{Mpc}^{-1}\right)$, which corresponds to a spatial scale of $1^{\prime \prime}=450 \mathrm{pc}$.

\section{OBSERVATIONS AND REDUCTIONS}

\subsection{HST WFPC2 Imaging}

We observed NGC 5252 with the Wide Field and Planetary Camera 2 (WFPC2) of HST through several narrowband and broadband filters in order to study the fine-scale morphology of the line-emitting gas near the nucleus and farther out within the bicone. Most of these images- $\mathrm{H} \alpha+$ [N II] $\lambda \lambda 6548,6583$, [O II] $\lambda 3727$, and [Ne v] $\lambda \lambda 3346,3426$ emission-line images and a line-free continuum image near $5900 \AA$-were presented by TMWC. Here we discuss a new WFPC2 image taken on 1995 July 23 through the linear ramp filter (LRF) FR533N, which isolates the redshifted [O III] $\lambda 5007$ emission line. This [O III] line is the brightest emission line in the optical spectrum and traces fainter filaments than were visible in the $\mathrm{H} \alpha+[\mathrm{N} \mathrm{II}]$ image. In addition, comparing the $[\mathrm{O} \mathrm{III}]$ and $\mathrm{H} \alpha+[\mathrm{N}$ II] images allows us to map spatial variations in the gaseous excitation and extinction of the line-emitting filaments.

To obtain the [O III] HST image, we placed the nucleus of NGC 5252 on the WF2 chip at pixel position $(278,219)$. The spacecraft roll orientation was such that the direction of the gradient in the LRF central wavelength coincided roughly with the axis of the ionization bicone. With an approximate bandpass $\delta \lambda / \lambda \approx 1.3 \%$ for the FR533N LRF, all [O III] $\lambda 5007$ emission within $\sim 10^{\prime \prime}$ of the nucleus was included in the image; emission at larger radii was attenuated by the spatially varying bandpass of the LRF. The total exposure was $2 \times 1200 \mathrm{~s}$.

We followed the data reduction procedures outlined in TMWC (see also Heathcote et al. 1996) to process the WFPC2 images. Briefly, standard STSDAS/WFPC2 pipeline files and procedures were used for bad pixel masking, analog-to-digital correction, and bias subtraction. Then, using by-hand methods described in Holtzman et al. (1995), we corrected remaining low-level bias structure and "hot" pixels using suitable "superbias," "superdark," and "deltadark" frames obtained from the WFPC2 instrument team. We used the standard WFPC2 F547M flat-field frame- the most suitable for the wavelength we observed - to correct for pixel-to-pixel sensitivity and low-frequency response variations in the region near the nucleus. Finally, flux calibration was achieved using the standard techniques outlined in the WFPC2 Instrument Handbook (Biretta et al. 1996), except that we estimated the total system throughput of the FR533N filter by comparing the continuum flux transmitted to that observed through the F588N filter (see TMWC), which has a known throughput. Applying a color correction of 0.3 magnitudes (appropriate for a typical S0/E galaxy) to account for the difference between the central wavelengths of the two filters, we adopted a system throughput for the FR533N filter of $\sim 4.5 \%$. This throughput is consistent with the ratio of the published filter transmissions in the F502N and FR533N filters, where the color correction is minimal. We removed the continuum from the [O III] image by aligning, scaling, and subtracting the F588N image, as described in TMWC.

We first used the STSDAS task WMOSAIC to scale all of our WFPC2 images to a common pixel scale of 0"1 pixel $^{-1}$ and to correct image distortions. Then we rotated and registered all of our WFPC2 images in order to make detailed comparisons. We registered the emission-line images by assuming that both the middle blob of the three colinear nuclear clumps and a point source $\sim 22^{\prime \prime}$ north of the nucleus (see TMWC and the present Fig. 1) are coincident in the various lines. The peak of the continuum image was also assumed to coincide with the position of the middle nuclear emission blob. ${ }^{4}$

\subsection{Fabry-Perot Velocity Maps}

We mapped the velocity field of the extended lineemitting gas in NGC 5252 using the Rutgers/Cerro Tololo Inter-American Observatory (CTIO) imaging Fabry-Perot (F-P) spectrometer on the CTIO $4 \mathrm{~m}$ telescope on 1994 April 1. Observations were made in the light of redshifted [O III] $\lambda 5007$. The F-P was configured with a camera lens of $135 \mathrm{~mm}$ focal length and a Tek1K CCD, yielding a field of diameter $\sim 2.5$ and a spatial scale of $\sim 0$ ".36 pixel $^{-1}$. We used the Rutgers "narrow" etalon, which has a spectral

\footnotetext{
${ }^{4}$ The $H S T$ absolute astrometry contained in the image headers could not be used to align the images because there was a 1 ."4 discrepancy between the right ascensions and declinations of both the middle nuclear blob and the off-nuclear point source in the $\mathrm{H} \alpha+[\mathrm{N}$ II $]$ image (taken on the PC1 CCD) compared with the [O III] image (taken on the WF2 CCD). The discrepancy results from using different guide stars and different spacecraft orientations during the observations on the different chips.
} 

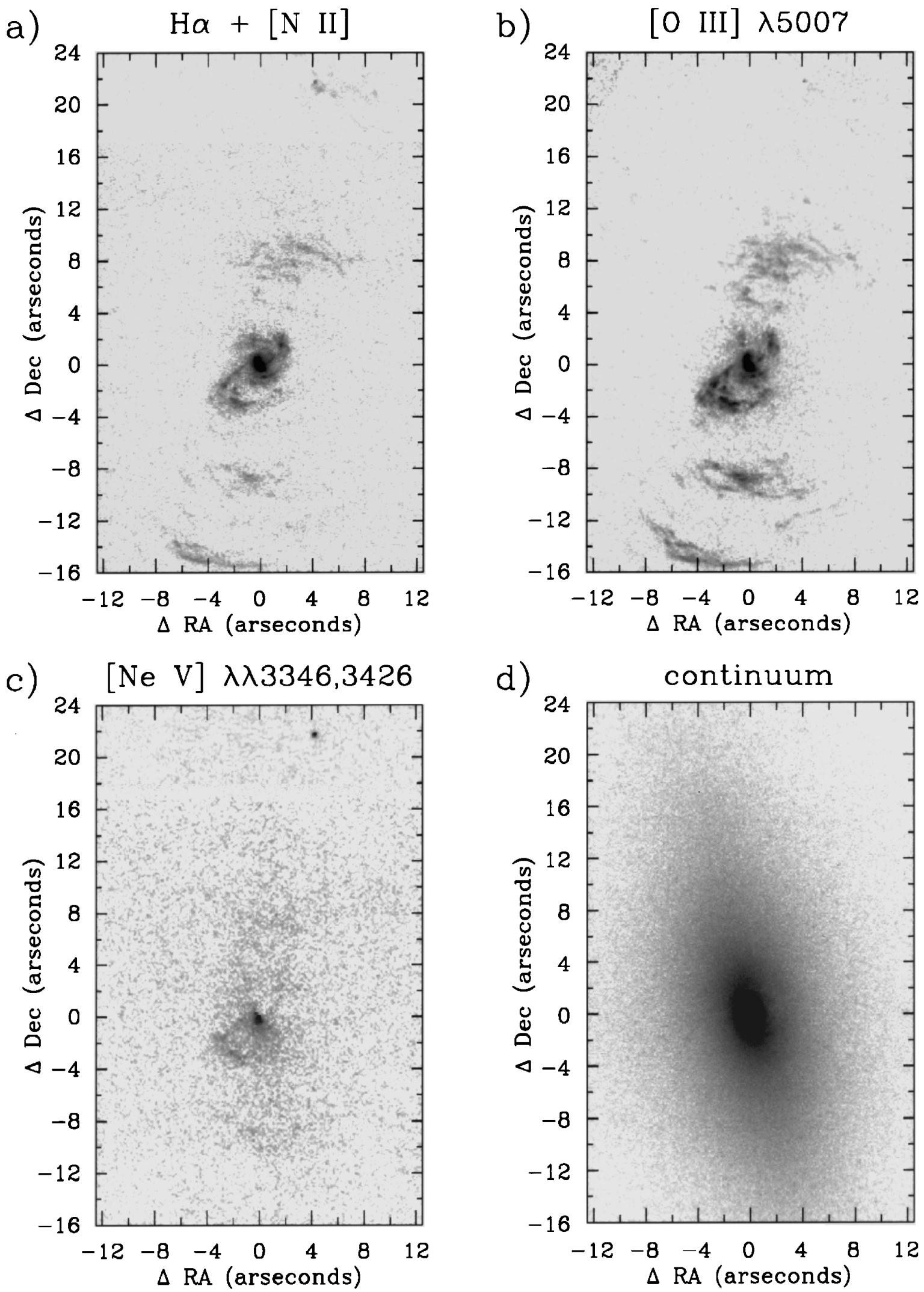

FIG. 1.-WFPC2 images of NGC 5252 in (a) H $\alpha+[\mathrm{N} \mathrm{II}] \lambda \lambda 6548,6583$ (F673N), (b) [O III] $\lambda 5007$ (FR533N), (c) [Ne v] $\lambda \lambda 3346,3426$ (F336W), and (d) yellow continuum (F588N). North is to the top and east is to the left. Intensities are shown with a logarithmic stretch in the "negative" images displayed. The middle blob of the three collinear nuclear clumps (see TMWC) is roughly at position ( $\Delta$ R.A., $\Delta$ decl. $\left.=0^{\prime \prime}, 0^{\prime \prime}\right)$ in each emission-line image; we also assumed that the peak of the continuum image coincides with the middle clump. The off-nuclear point source is at position $\left(4^{\prime \prime}, 22^{\prime \prime}\right)$. The plots are truncated in the south because of the limited field of view of the planetary camera in $(a)$ and $(c)$ and the sloping bandpass of the LRF in $(b)$. 
resolution of $\sim 30 \mathrm{~km} \mathrm{~s}^{-1} \mathrm{FWHM}$ and a free spectral range of $\sim 13.5 \AA\left(790 \mathrm{~km} \mathrm{~s}^{-1}\right)$ at $5125 \AA$. The [O III] emissionline profiles were Nyquist-sampled with a sequence of $600 \mathrm{~s}$ exposures at $15 \mathrm{~km} \mathrm{~s}^{-1}$ intervals over a total velocity range of $210 \mathrm{~km} \mathrm{~s}^{-1}$, centered on the galaxy systemic heliocentric velocity $V_{\text {sys }}=6925 \mathrm{~km} \mathrm{~s}^{-1}$. We chose this range based on the radial-velocity observations of Held, Capaccioli, \& Cappellaro (1992, hereafter HCC). The images at the most extreme velocities did not contain extended line emission, so these were summed and used to subtract the stellar continuum from the other images.

The redshift of NGC 5252 shifts the [O III] $\lambda 5007$ line to $\sim 5122 \AA$. We placed a narrowband filter centered at $\lambda_{\mathrm{c}}=$ $5125 \AA$ with a FWHM of $\delta \lambda=44 \AA$ in the converging telescope beam above the F-P. This filter passed $\sim 3$ spectral orders at each point in the galaxy, isolating the [O III] $\lambda 5007$ emission line from neighboring lines but allowing three times more continuum flux than from a single order. The filter was tilted to ensure that ghost images did not fall on the CCD. In addition, we placed the galaxy off-center in our field of view to avoid confusion with the antisymmetric $\mathrm{CCD} /$ talon ghost. The seeing, as determined from radial brightness profiles of a faint star in the images, was generally $\sim 1$ ".4 FWHM, though ranged between $\sim 1$ ".2 and 1".8 FWHM. Drifts in the wavelength zero point due to temperature variations in the etalon were monitored hourly by obtaining exposures of the $\mathrm{Ne}$ I $\lambda 5113.8$ line in a calibration lamp.

We processed the F-P data using our standard procedures (Morse et al. 1992, 1993; see also Atherton et al. 1982 and Bland \& Tully 1989 for more detailed discussions). The images were cleaned of cosmic rays, biassubtracted, corrected for atmospheric extinction using standard CTIO extinction coefficients, and assembled sequentially into a velocity "data cube." A corresponding "white light" cube of dome flat fields, taken at every etalon gap setting for which galaxy data were obtained, was used to correct for pixel-to-pixel sensitivity variations and the transmission profile of the order-separating filter. We summed these flat-field frames to improve signal statistics, smoothed the resulting "superflat" with a broad Gaussian function, and then divided the unsmoothed by the smoothed image. Each image of the data cube was next divided by this normalized superflat to remove pixel-topixel variations. The images of the white light cube at the individual etalon settings were then smoothed with the same broad Gaussian function. The resulting smoothed white light cube was normalized to the maximum of the filter transmission profile and divided into the velocity data cube to remove modulations in the filter response at each pixel. Individual frames in the data cube were registered using the galaxy nucleus and a faint star $\sim 65^{\prime \prime}$ southsoutheast of the nucleus as tie points. Finally, the data cubes were phase-corrected to stacks of monochromatic images (see Bland \& Tully 1989).

\section{EMPIRICAL RESULTS}

\subsection{Small-Scale Structure near the Nucleus}

Figure 1 shows a mosaic of our HST WFPC 2 images of the circumnuclear region of NGC 5252. The region shown is $25^{\prime \prime} \times 40^{\prime \prime}$ and is truncated, especially to the south, by the field of view afforded by the Planetary Camera (PC) and by the wavelength gradient of the LRF in the [O III] image.
In fact, as mentioned earlier, the [O III] emission from the northernmost arcs shown is significantly attenuated by the filter bandpass.

TMWC have discussed in detail the morphology of the ionized gas within $5^{\prime \prime}(2.2 \mathrm{kpc})$ of the nucleus. The line emission in the inner $1^{\prime \prime}(450 \mathrm{pc})$ concentrates in three bright collinear blobs. The middle and brightest of the three blobs appears to be the nucleus, or lies very close to it (within $0^{\prime \prime} .2$ ). This blob is essentially pointlike in our PC images, and HST Faint Object Spectrograph (FOS) spectra show that it has much broader line profiles than the two clumps bracketing it (Tsvetanov et al. 1998). The outer two blobs have filamentary extensions in the PC images. The finescale morphology of the three nuclear clumps is not shown as well in the $0^{\prime \prime} .1$ pixel $^{-1}$ images presented here, and the interested reader is referred to TMWC for details.

On larger scales, the line-emitting gas is distributed in two major and several smaller "spiral arms" wound counterclockwise that extend $\sim 3^{\prime \prime}(1.3 \mathrm{kpc})$ to the northwest and $\sim 4 " .5(2.0 \mathrm{kpc})$ to the southeast. These spiral arms and other arcuate features in our field of view are seen particularly well in the $\mathrm{H} \alpha+[\mathrm{N} \mathrm{II}]$ and [O III] images (Fig. 1; see also Fig. 2 of TMWC). The detailed substructure and changes in line ratios within the spiral arms are depicted in the upper right-hand panel of Figure 2, which shows a falsecolor composite of the three emission-line images from Figure 1 . Here the $[\mathrm{Ne} \mathrm{v}]$ image is blue, $[\mathrm{O} \mathrm{III}]$ is green, and $\mathrm{H} \alpha+[\mathrm{N} \mathrm{II}]$ is red. The southeast arm contains knots and filaments $0.2-0$ ".5 ( 90-225 pc) in size and is brightest in [O III]. In contrast, in the northwest arm, some filaments that are prominent in $\mathrm{H} \alpha+[\mathrm{N} \mathrm{II}]$ are very faint in [O III]. The $[\mathrm{O} \mathrm{III}] /(\mathrm{H} \alpha+[\mathrm{N} \mathrm{II}])$ ratios, shown in the lower right inset of Figure 2, thus tend to be high in the southeast arm and low in the northwest arm.

The arcs of emission beyond $5^{\prime \prime}(2.2 \mathrm{kpc})$ radius usually resolve into separate strands that overlap in a complicated fashion. These strands are typically $0.2-0$ ".3 (90-135 pc) wide but may be several arcseconds long. We show below that some extended filaments that appear adjacent on the sky actually have rather different kinematics; such is the case for the group of strands $\sim 8^{\prime \prime}-10^{\prime \prime}(3.6-4.5 \mathrm{kpc})$ north of the nucleus (see Figs. $1 a$ and $b$ ). There is also a suggestion that filaments with different kinematics have different curvatures. These observations taken together may indicate the presence of distinct gaseous structures at different locations within the ionization bicone but projected close to each other on the sky.

An unresolved radio source $\sim 22^{\prime \prime}$ north of the nucleus of NGC 5252 (see WT94; TMWC) is detected in all three emission-line images of Figure 1 and perhaps marginally in the $\mathrm{F} 588 \mathrm{~N}$ continuum image. The mysterious nature of this source is not clarified by our new FR533N image, wherein the point source is faint. It could be either a continuum source (within NGC 5252 or foreground/background to it) or an emission-line object associated with the galaxy. However, the very blue color of the source (Figs. 1 and 2) suggests it may be a background quasar, in which case it may contribute significant $2-10 \mathrm{keV}$ flux to the $A S C A$ X-ray spectrum of NGC 5252 reported by Cappi et al. (1996).

\subsection{Large-Scale Morphology and Kinematics}

Figures 3 and 4 summarize our F-P data. Figure 3 shows a mosaic of [O III] F-P velocity slices spanning the total 


\section{NGC 5252 - HST WFPC2}
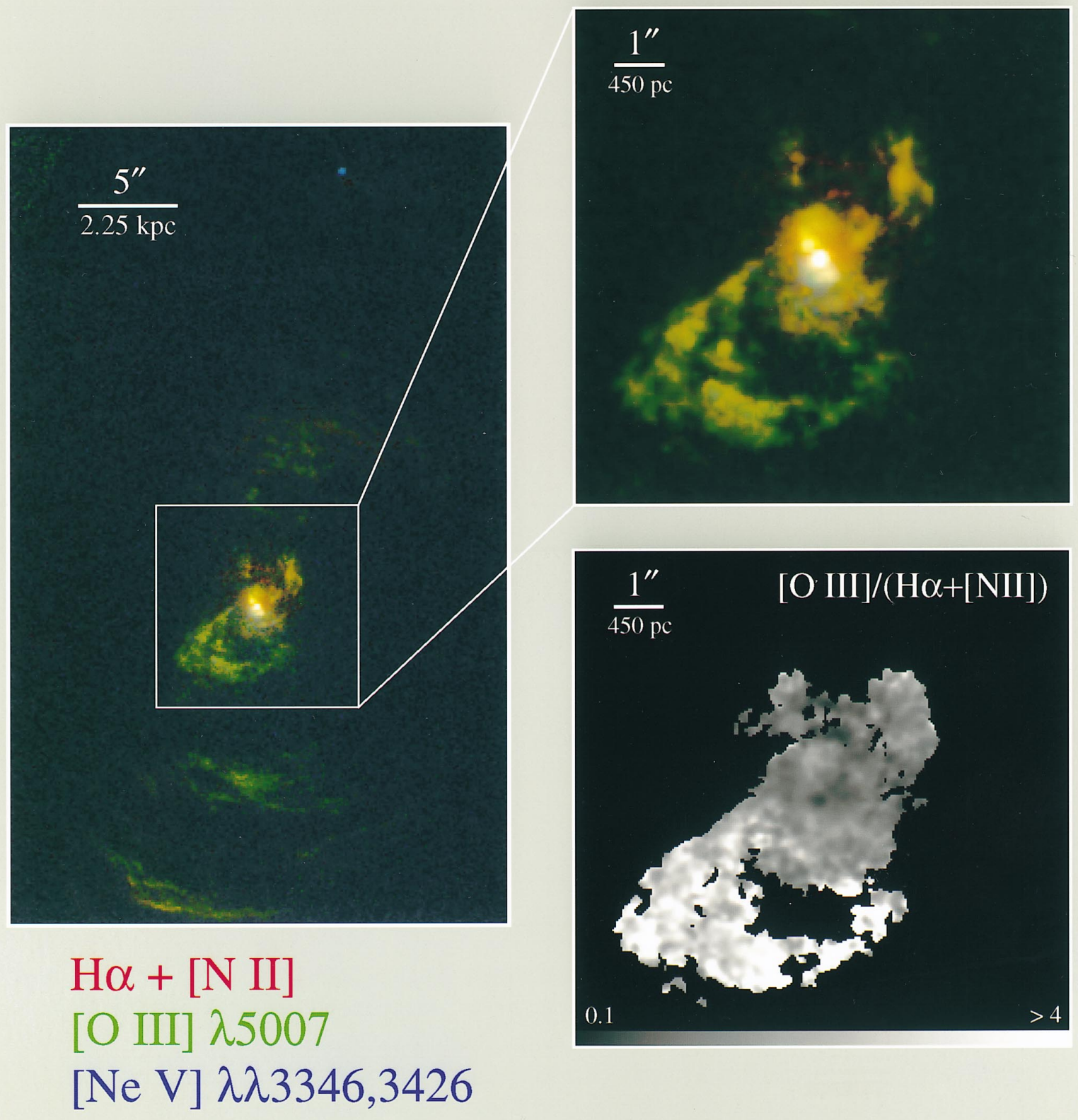

FIG. 2.-Color composite images of the line emission from the ionized gas surrounding the nucleus of NGC 5252. The composite was made by combining the flux-calibrated emission-line images from Fig. 1, with $\mathrm{H} \alpha+[\mathrm{N} \mathrm{II}]$ in red, [O III] in green, and [Ne v] in blue. The left-hand panel shows the same region as Fig. 1, while the upper right-hand panel magnifies the central 10" square region. Most of the extended emission arcs and clumps are dominated by [O III] emission. However, the three nuclear blobs and several filaments in the northwest spiral arm are relatively bright in $\mathrm{H} \alpha$. The lower right-hand panel displays these trends in the line ratios, with lighter shades representing higher $[\mathrm{O} \mathrm{III}] /(\mathrm{H} \alpha+[\mathrm{N} \mathrm{II}])$ ratios. A minimum in the line ratio occurs at the position of the middle nuclear blob. The off-nuclear point source near the top of the left-hand image appears very blue because of its brightness in the [Ne v] (F336W) image. It is unclear at this time whether the point source is a blue continuum source or an emission-line object. 


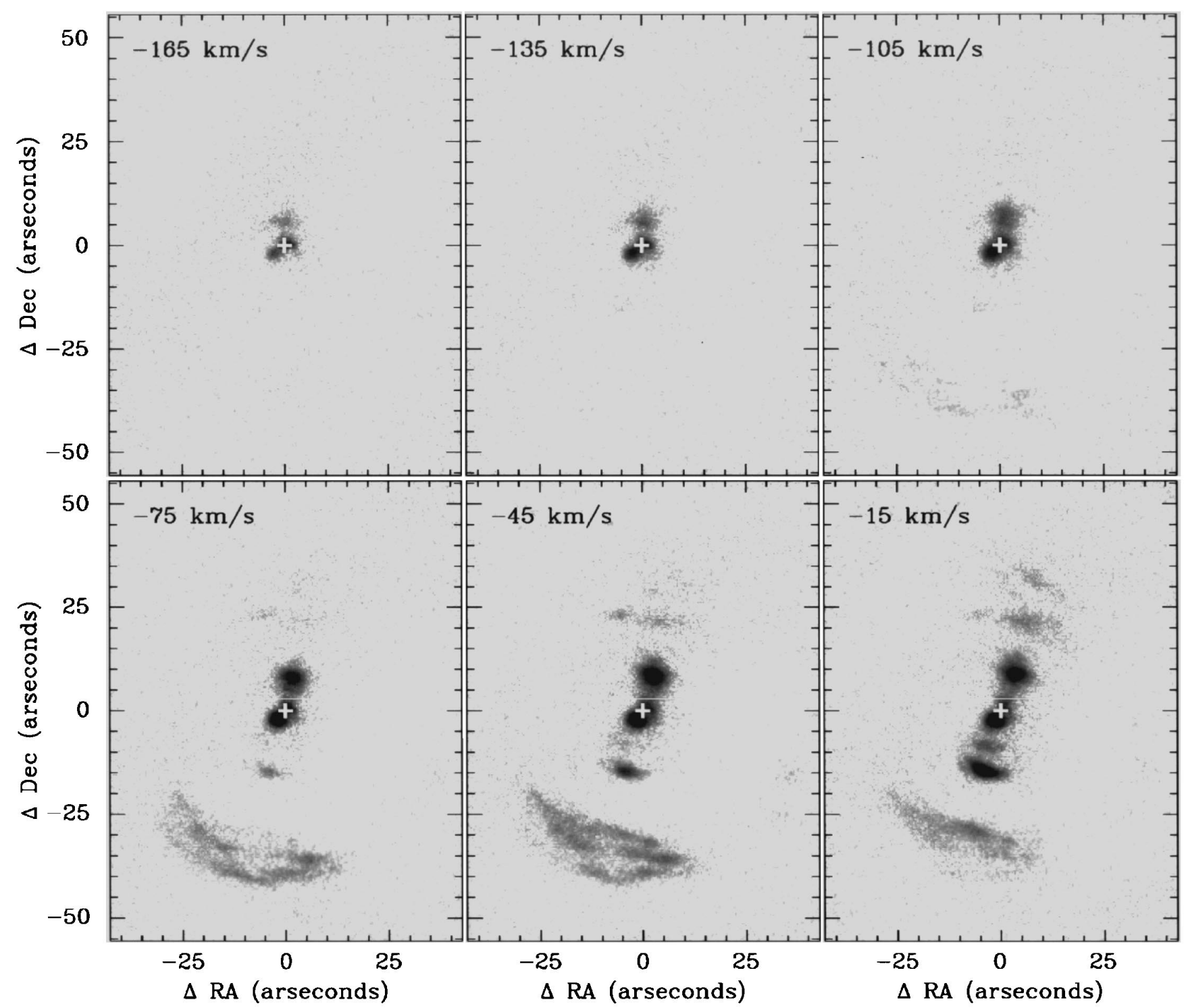

FIG. 3. - Subset of the individual, continuum-subtracted [O III] $\lambda 5007$ velocity slices of NGC 5252 from our F-P data cube. The velocities indicated are relative to the systemic velocity of the galaxy $\left(V_{\text {sys }}=6925 \mathrm{~km} \mathrm{~s}^{-1}\right)$. A white cross marks the approximate position of the nucleus in each panel. The shrinking circular background seen in the most redshifted slices is an artifact of the phase correction. Although the detailed morphology of the individual arcs of emission is different, a stark antisymmetry in the velocity field across the nucleus is evident (see also Fig. 4).

velocity range of the extended line-emitting gas. The velocities indicated in each panel are relative to the systemic velocity of the galaxy $\left(V_{\text {sys }}=6925 \mathrm{~km} \mathrm{~s}^{-1}\right.$; Huchra \& Burg 1992). The mosaic highlights an obvious antisymmetry of the velocity field of the ionized gas across the nucleus but also clearly reveals that both blueshifted and redshifted features are found on each side of the nucleus (see also HCC). We also see that emission arcs projected next to each other can have quite different kinematics. In general, the highest gaseous velocities with respect to systemic are seen relatively close to the nucleus.

The left panel of Figure 4 displays the logarithm of the [O III] $\lambda 5007$ emission intensity summed over the individual velocity slices (equivalent to a narrow-band [O III] image) and indicates the large-scale morphology of the extended ionized gas. This image shows the full ionization bicone, discovered by TT89, extending $\pm 45^{\prime \prime}-50^{\prime \prime}$ from the nucleus. The bicone has an apparent opening angle of $\sim 75^{\circ}$, and its axis lies along P.A. $\sim 163^{\circ}$, misaligned with the photometric major axis of the galaxy by $\sim 30^{\circ}$. The emission is distributed in a series of arcs, some of which resolve into complex networks of strands in the HST images (Figs. 1 and 2).

The right-hand panel of Figure 4 depicts in color the Doppler velocities of the gas relative to the systemic velocity of the galaxy. ${ }^{5}$ Outside the nuclear region, the deepest blue and red colors show that the highest gaseous velocities $\left( \pm>100 \mathrm{~km} \mathrm{~s}^{-1}\right)$ are found in the circumnuclear spiral arms within $\sim 5^{\prime \prime}$ of the nucleus (Fig. 1) and in regions

\footnotetext{
${ }^{5}$ The thin bands of blue and red right at the continuum peak (cross) reflect the double-peaked line profiles at the position of the nucleus (e.g., Acosta-Pulido et al. 1996). This region, within $\sim 1^{\prime \prime}-2^{\prime \prime}$ of the nucleus, is spatially unresolved in the ground-based F-P data. We use HST FOS spectra and a decomposition of the F-P line profiles to study the nuclear region in a later paper (Tsvetanov et al. 1998).
} 


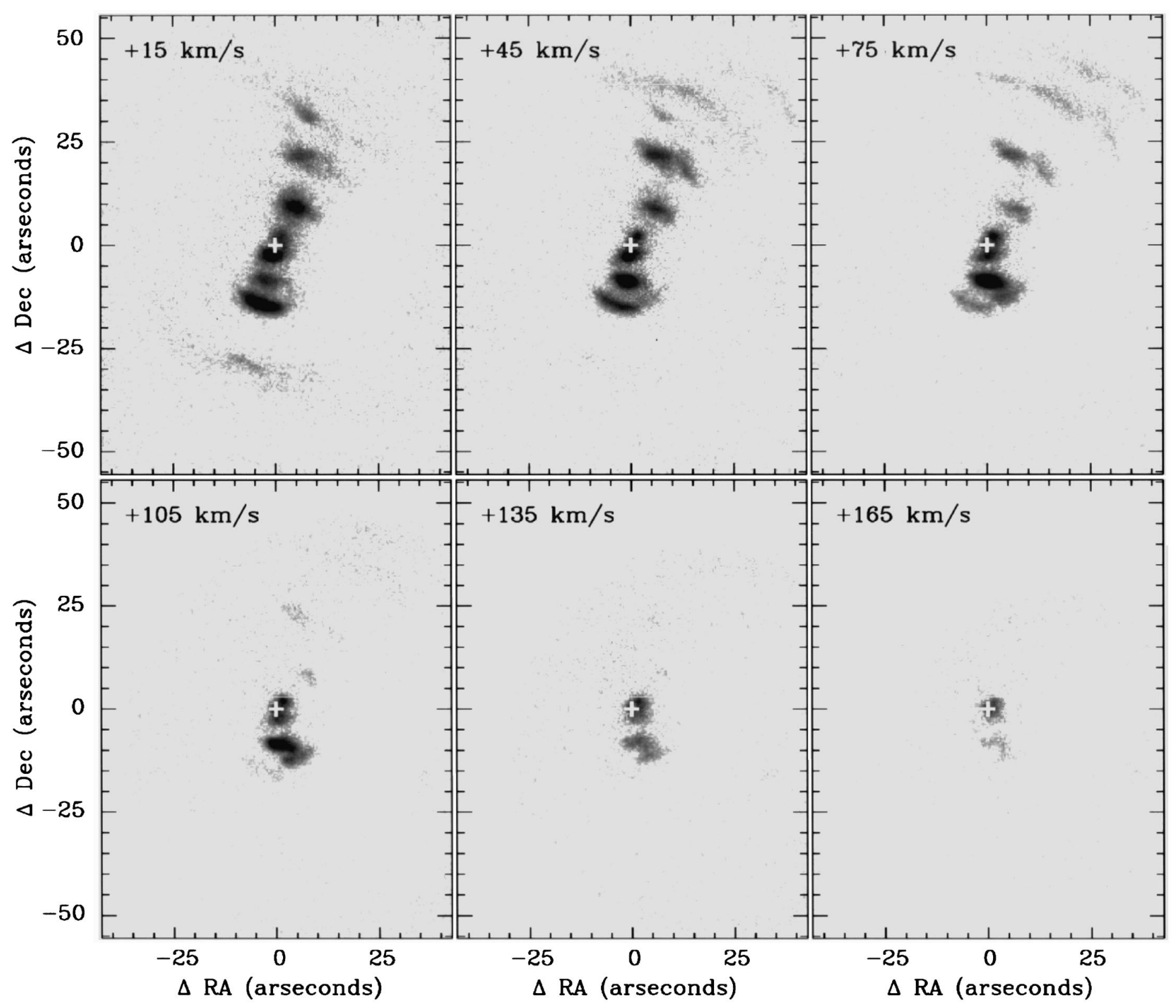

Fig. 3.-Continued

located $5^{\prime \prime}-15^{\prime \prime}$ north and south of the nucleus (see also Fig. 3). Gas velocities in the latter regions are comparable to the stellar velocities, but of opposite sign (see HCC).

In the line-flux image (Fig. 4), the circumnuclear spiral arms are the emission blobs immediately northwest and southeast of the cross. The spiral structure evident in the HST images is not resolved, but the general morphology follows that seen in the WFPC2 images (Fig. 1). In the velocity map, the northwest arm is redshifted, and the southeast arm is blueshifted, as previously noted by TMWC. The southeast arm also shows a velocity gradient, with velocities increasing from east to west.

The bicone defined by the most extended emission arcs (e.g., the northern cone seen in the $+45 \mathrm{~km} \mathrm{~s}^{-1}$ slice and the southern cone in the $-45 \mathrm{~km} \mathrm{~s}^{-1}$ slice in Fig. 3) does not contain all of the line emission. In particular, the circumnuclear spiral arms, especially the one to the southeast, extend outside the cone edges and seem almost to surround the nucleus (Fig. 2). In the next section, we argue from the spatial distribution and kinematics of the ionized gas that more than one dynamical gaseous component exists in NGC 5252.
In Figure 5, we plot the space-velocity diagram of the extended ionized arcs, summed azimuthally at each radius. Arcs north of the nucleus are shown in red and arcs south of the nucleus in green. Large azimuthal gradients in velocity along some arcs, especially those within $\sim 10^{\prime \prime}$ of the nucleus, project to large velocity ranges at a given distance $R$ from the nucleus. This effect is particularly large in the circumnuclear spiral arms at $R<5^{\prime \prime}$. However, the large arcs beyond $\sim 20^{\prime \prime}$ from the nucleus have small projected velocity ranges. There is an obvious antisymmetry in the velocities to the north and south, although the brightest features on opposite sides of the nucleus sometimes interleave in projected distance.

\section{DISCUSSION}

\subsection{Physical Conditions in the Line-emitting Filaments}

The morphology of the circumnuclear spiral arms within $\sim 5^{\prime \prime}$ of the nucleus, seen clearly in the HST [O III] and $\mathrm{H} \alpha+[\mathrm{N} \mathrm{II}]$ emission-line images (Fig. 1), strongly suggests a rotating gas disk (TMWC). Consistent with this, the velocities relative to systemic of the arms on opposite sides 


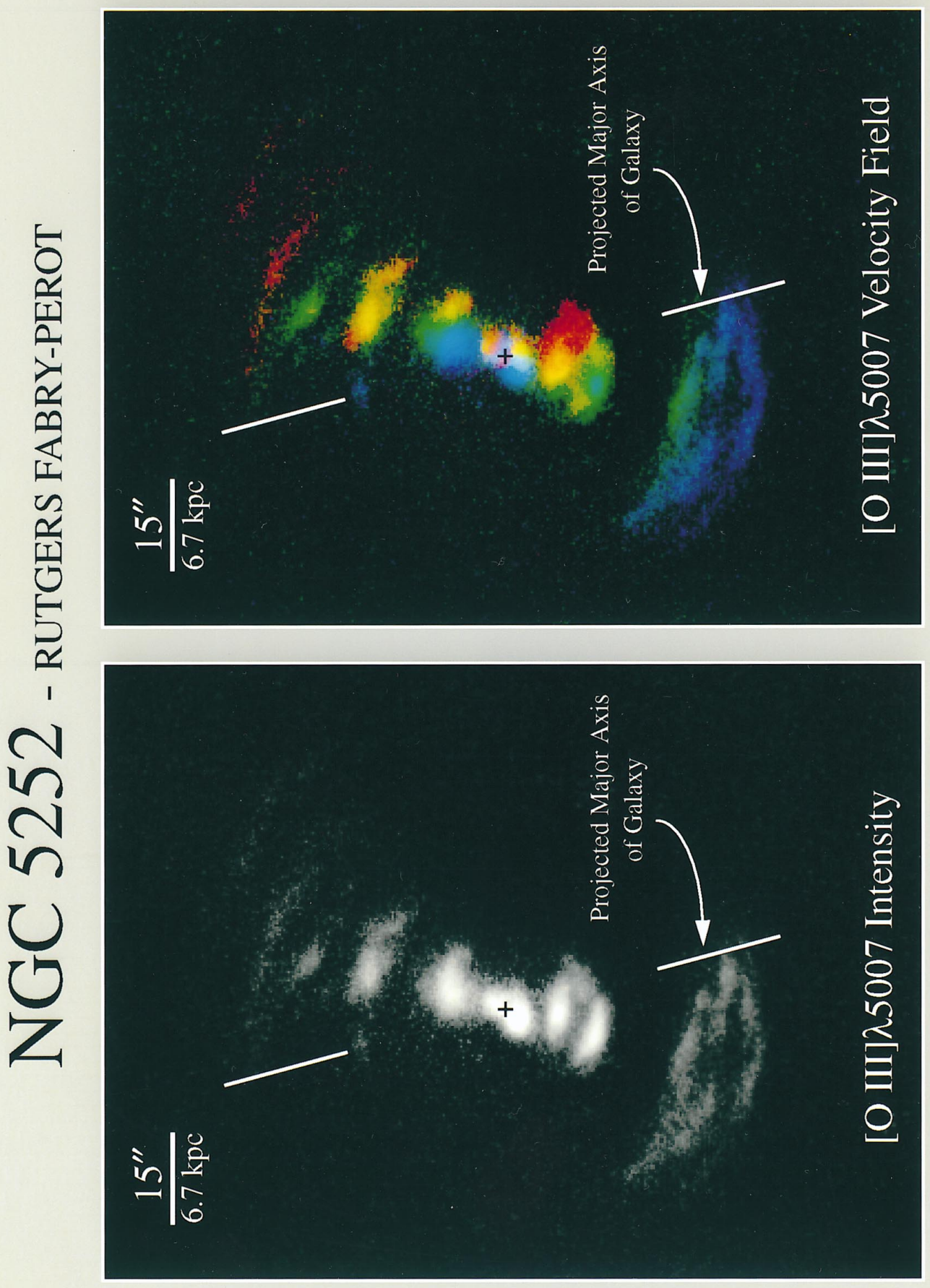

긍 중

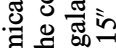

至要焉

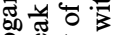

으 흐를 증

형응

प्र०

ธั้ 흘 ही

氙需范

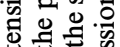

$\exists$ 요웅

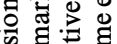

䗆

हैं 원 0

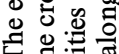

ज陆

ठํ

ช त् 气े

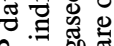

它政

$\exists$ 웡

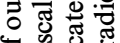

그음

क्ष

폴

혹웜

它

크월

<交。

웜

on

폼. 핑

㝕

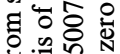

주

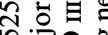

엻을 數 政 ヨ.

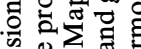
贻

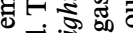

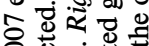
号它. 目然要要 을 0 80 ज行

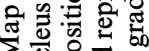
$\sum$ 융 菏矛至 त त.

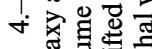

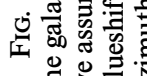
$\approx$ 응 


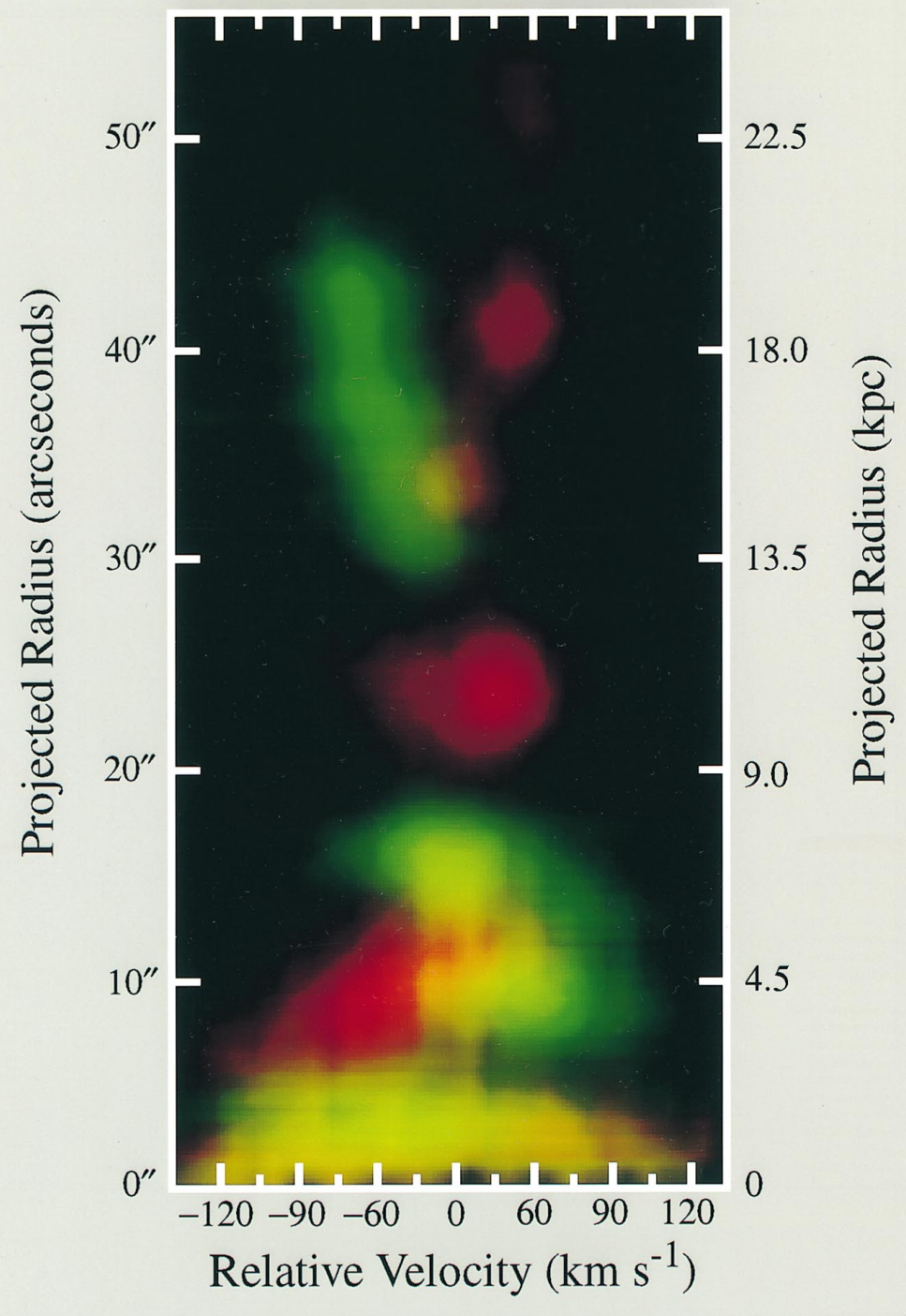

FIG. 5.-Radial velocity field of the ionized gas in NGC 5252 projected to the $R-V$ plane, where $R$ is the radial distance from the nucleus and $V$ is the velocity with respect to the galaxy systemic. Emission from structures north of the nucleus is colored red, and emission from south of the nucleus is colored green.

of the nucleus are roughly equal in magnitude and of opposite sign, and there is a large azimuthal gradient of velocity along the southeast arm (Figs. 3 and 4).

TMWC showed that the gas disk containing the circumnuclear spiral arms has a projected major axis of P.A. $\sim 135^{\circ}$ and, assuming the disk is intrinsically circular, is inclined $\sim 40^{\circ}$ to the line of sight. If the arms trail, the gas disk rotates clockwise on the sky, with the northeast side being the near side. Based on the obscuration pattern presented by TMWC, in which the filaments in the northwest spiral arm are also seen in absorption against the background starlight, the stars lie behind the northwest arms and in front of the southeast arms. TMWC also estimated that the neutral hydrogen column density through the fila- 
ments is $N_{\mathrm{H}} \sim 5 \times 10^{20} \mathrm{~cm}^{-2}$, assuming the standard Galactic extinction law and a normal gas-to-dust ratio.

TMWC calculated that the $\mathrm{H} \alpha$ flux and the extinction observed in the filaments in the northwest spiral arms imply ionized and neutral hydrogen volume densities of a few $\mathrm{cm}^{-3}$. In this section, we present a more extensive discussion of the physical conditions in the ionized gas in NGC 5252.

We begin by deriving the density in the southeast arms of the circumnuclear spiral in a similar manner to that used by TMWC. Assuming case $\mathrm{B}$ conditions, the $\mathrm{H} \alpha$ luminosity is

$$
L_{\mathrm{H} \alpha}=j_{\mathrm{H} \alpha} V f \operatorname{ergs~s}^{-1},
$$

with $V$ the total volume of the emitting cloud or filament, $j_{\mathrm{H} \alpha}$ the volume emissivity, and $f$ the volume-filling factor of the gas responsible for the line emission. For an electron temperature in the $\mathrm{H} \alpha$-emitting gas of $T=10^{4} \mathrm{~K}$,

$$
j_{\mathrm{H} \alpha}=3.6 \times 10^{-25} n_{p} n_{e} \operatorname{ergs} \mathrm{cm}^{-3} \mathrm{~s}^{-1},
$$

where $n_{p}$ is the number density of protons, which we approximate to be $\sim 0.9$ times the number density of electrons, $n_{e}$. Combining the above equations leads to the expression

$$
n_{e}=1.8 \times 10^{12}\left(\frac{L_{\mathrm{H} \alpha}}{V f}\right)^{1 / 2} \mathrm{~cm}^{-3} .
$$

For a bright cloud in the southeast arm, which we approximate as a sphere of radius $r \sim 90 \mathrm{pc}\left(00^{\prime \prime} .2\right)$, the measured $\mathrm{H} \alpha$ flux from our WFPC2 image is $F_{\mathrm{H} \alpha} \approx 8.3 \times 10^{-16}$ ergs cm $\mathrm{cm}^{-2} \mathrm{~s}^{-1}$, which corresponds to a luminosity of $L_{\mathrm{H} \alpha} \approx$ $8.4 \times 10^{38}$ ergs s$^{-1}$ after correcting the $\mathrm{H} \alpha$ flux and luminosity for contamination by the [N II] $\lambda \lambda 6548,6584$ lines in the manner described in TMWC. With a volume of $V \approx 10^{62} \mathrm{~cm}^{3}$, the number density of ionized gas is $n_{e} \sim$ $5 /(f)^{1 / 2} \mathrm{~cm}^{-3}$.

The total mass of ionized gas, $M_{\text {ion }}$, considering the contributions of hydrogen and helium only, is

$$
M_{\text {ion }}=\left(n_{p} m_{p}+n_{\mathrm{He}} m_{\mathrm{He}}\right) V f \mathrm{~g},
$$

which, using the approximations $n_{\mathrm{He}} \approx 0.1 n_{p}$ and $n_{e} \approx$ $\left(n_{p}+1.5 n_{\mathrm{He}}\right)$, reduces to

$$
\begin{aligned}
M_{\text {ion }} & =1.2 n_{e} m_{p} V f \mathrm{~g} \\
& =1 \times 10^{-57} n_{e} V f M_{\odot} .
\end{aligned}
$$

Using the electron density and volume calculated above, the mass of ionized gas in the southeast arm cloud is $M_{\text {ion }}=5 \times 10^{5}(f)^{1 / 2} M_{\odot}$.

The number of recombination photons observed per second, $Q_{\text {rec }}$, is

$$
\begin{aligned}
Q_{\mathrm{rec}} & =\left(\frac{\alpha_{B} L_{\mathrm{H} \alpha}}{\alpha_{\mathrm{H} \alpha} h v_{\mathrm{H} \alpha}}\right) \\
& =7.3 \times 10^{11} L_{\mathrm{H} \alpha} \text { photons s }^{-1},
\end{aligned}
$$

where $h v_{\mathrm{H} \alpha}$ is the energy of an $\mathrm{H} \alpha$ photon, and we have used $\alpha_{B} / \alpha_{\mathrm{H} \alpha}=2.2$. Then $Q_{\mathrm{rec}}=6.6 \times 10^{50}$ photons $\mathrm{s}^{-1}$ for the southeast arm cloud. Using the geometry of the spiral disk and dimensions of the cloud cited above, the cloud is $R \sim 1700 \mathrm{pc}$ from the nucleus and subtends a solid angle on the sky of $\Omega \sim 8.8 \times 10^{-3} \mathrm{sr}$ as seen from the nucleus. Assuming that the gas is ionized by a point source at the nucleus and that this source radiates isotropically, the number of ionizing photons emitted is $Q_{\text {ion }}=9.4 \times 10^{53} \epsilon^{-1}$ photons $\mathrm{s}^{-1}$, where $\epsilon$ is the fraction of ionizing photons incident on the cloud that are absorbed. The ionization parameter $\Gamma$ (the ratio of the ionizing photon density at the cloud to the electron density) is

$$
\Gamma=\frac{Q_{\text {ion }}}{4 \pi R^{2} c n_{e}},
$$

where $c$ is the speed of light. Inserting the above values, we find $\Gamma \approx 1.8 \times 10^{-2} \epsilon^{-1} f^{1 / 2}$. The values we have derived for $n_{e}, M_{\text {ion }}, Q_{\text {rec }}$, and $Q_{\text {ion }}$ should be considered lower limits, since we have not corrected the observed $\mathrm{H} \alpha$ flux for reddening; even a modest correction of $E(B-V) \sim 0.1$ will increase these values by $>12 \%-25 \%$. Also, modest extinction between the nucleus and the cloud will increase $Q_{\text {ion }}$; such extinction is likely in a thin disk geometry.

We can also estimate the density of ionized gas from the flux of the $\left[\begin{array}{ll}\mathrm{O} & \mathrm{III}\end{array}\right] \mathrm{5007}$ forbidden-line emission (Osterbrock 1989). The volume emissivity $j_{4959,5007}$ of the [O III] $\lambda \lambda 4959,5007$ lines is related to the number density of doubly ionized oxygen atoms in the excited state $n_{2}$ and in the ground state $n_{1}$, within the [O III]-emitting region of the ionized filaments, by the equation

$$
j_{4959,5007}=n_{2} A_{21} h v_{21}=n_{1} n_{e} q_{12} h v_{21} \operatorname{ergs~} \mathrm{cm}^{-3} \mathrm{~s}^{-1} \text {, }
$$

where

$$
q_{12}=\frac{8.63 \times 10^{-6} \Upsilon_{12} e^{-\left(h v_{12} / k T_{e}\right)}}{g_{1} T_{e}^{1 / 2}} \mathrm{~cm}^{3} \mathrm{~s}^{-1}
$$

is the collisional excitation rate coefficient, $A_{21}$ is the total Einstein A-value for the transition, $\Upsilon_{12}$ is the collision strength, $g_{1}$ is the ground state statistical weight, and $T_{e}$ is the electron temperature. Equation (10) assumes that the electron density is much lower than the critical density $\left(n_{C} \approx 8.1 \times 10^{5} \mathrm{~cm}^{-3}\right)$ for this transition, which is almost certainly the case, given the density estimates based on the $\mathrm{H} \alpha$ flux. The total number $n_{1}$ of doubly ionized oxygen atoms in the ground state can be expressed in terms of the total number of atoms by

$$
n_{1}=\left[\frac{n_{1}}{n\left(\mathrm{O}^{++}\right)}\right]\left[\frac{n\left(\mathrm{O}^{++}\right)}{n(\mathrm{O})}\right]\left[\frac{n(\mathrm{O})}{n(\mathrm{H})}\right]\left[\frac{n(\mathrm{H})}{n_{\mathrm{TOT}}}\right] n_{\mathrm{TOT}},
$$

where $n\left(\mathrm{O}^{++}\right), n(\mathrm{O}), n(\mathrm{H})$, and $n_{\text {TOт }}$ are the number densities of doubly ionized oxygen atoms, oxygen atoms, hydrogen atoms, and total atoms in the region of interest, respectively. Equation (10) can then be written

$$
\begin{aligned}
j_{4959,5007} \approx & {\left[\frac{n_{1}}{n\left(\mathrm{O}^{++}\right)}\right]\left[\frac{n\left(\mathrm{O}^{++}\right)}{n(\mathrm{O})}\right]\left[\frac{n(\mathrm{O})}{n(\mathrm{H})}\right]\left[\frac{n(\mathrm{H})}{n_{\mathrm{TOT}}}\right] } \\
& \times n_{\mathrm{TOT}} n_{e}\left[\frac{8.63 \times 10^{-6} \Upsilon_{12} e^{-\left(h v_{12} / k T_{e}\right)}}{g_{1} T_{e}^{1 / 2}}\right] \\
& \times h v_{21} \text { ergs cm } \mathrm{cm}^{-1} .
\end{aligned}
$$

To a good approximation $\left[n_{1} / n\left(\mathrm{O}^{++}\right)\right] \approx 1$. We assume an electron temperature $T_{e} \sim 15,000 \mathrm{~K}$ in the [O III]-emitting region (Tadhunter et al. 1989; Morse et al. 1996). For cosmic abundances (e.g., Allen 1973), $[n(\mathrm{O}) /$ $n(\mathrm{H})] \approx 6.6 \times 10^{-4}$. We also approximate $\left[n(\mathrm{H}) / n_{\mathrm{TOT}}\right] \sim$ 0.9 and $n_{\text {Tот }} \sim n_{e}$. Using the ground state statistical weight $g_{1}=9$, collision strength $\Upsilon_{12}=2.30$ (Mendoza 1983), $h v_{21}=3.97 \times 10^{-12} \quad$ ergs, and $\eta=\left[n\left(\mathrm{O}^{++}\right) / n(\mathrm{O})\right]$, 
equation (13) reduces to $j_{4959,5007} \approx 6.3 \times 10^{-24} \eta n_{e}^{2}$ ergs $\mathrm{cm}^{-3} \mathrm{~s}^{-1}$. Using the familiar $3: 1$ ratio of [O III] $\lambda 5007 /$ $\lambda 4959$, we obtain

$$
j_{5007} \approx 4.7 \times 10^{-24} \eta n_{e}^{2} \text { ergs } \mathrm{cm}^{-3} \mathrm{~s}^{-1} .
$$

The observed $\lambda 5007$ volume emissivity is

$$
j_{5007}=\frac{4 \pi d^{2} F_{5007}}{f V} \mathrm{ergs} \mathrm{cm}^{-3} \mathrm{~s}^{-1},
$$

where $d=92 \mathrm{Mpc}$ is the distance to NGC 5252. The electron density can then be calculated from the observed $\lambda 5007$ flux via the relation

$$
\begin{aligned}
n_{e} & =4.7 \times 10^{11}\left(\frac{4 \pi d^{2} F_{5007}}{\eta f V}\right)^{1 / 2} \\
& =4.7 \times 10^{11}\left(\frac{L_{5007}}{\eta f V}\right)^{1 / 2} \mathrm{~cm}^{-3} .
\end{aligned}
$$

For an $[\mathrm{O} \mathrm{III}] \lambda 5007$ flux of $F_{5007}=3.8 \times 10^{-15} \mathrm{ergs}$ $\mathrm{cm}^{-2} \mathrm{~s}^{-1}$ observed from the same cloud in the southeast arm as considered above, the calculated electron density $n_{e}=3 /(\eta f)^{1 / 2}$. This density estimate is comparable to that derived from the $\mathrm{H} \alpha$ flux, as long as $\eta \approx 1$. The density calculated from the $[\mathrm{O}$ III $] \lambda 5007$ flux would be larger if a modest correction for extinction were introduced, or if the oxygen abundance were smaller than assumed here.

The electron density estimates for the northwest and southeast circumnuclear spiral arm filaments are thus comparable. However, the ratio $[\mathrm{O} \mathrm{III}] /(\mathrm{H} \alpha+[\mathrm{N} \mathrm{II}])>1$ in the southeast arm, but $\lesssim 1$ in the northwest arm. This gradient, if due to extinction, is in the same sense as the map of dust obscuration obtained by TMWC (their Fig. $2 b$ ). In addition, although our F336W image shown in Figure 1 has low signal-to-noise ratios, the [ $\mathrm{Ne} \mathrm{v}]$ emission, which would be even more seriously affected by extinction, is seen only from the southeast arm and nuclear clumps, but not at all from the northwest arm. The observed gradients suggest both continuum and lines are reddened preferentially to the northwest, but it is not obvious why. TMWC argued that the continuum obscuration to the northwest implies that the stellar disk of the galaxy lies behind the gas disk, so that dust in the gas disk can obscure the background starlight. But if dust residing in the gas disk also reddens the line emission, we would expect to observe similar reddening effects on the $[\mathrm{O}$ III $] /(\mathrm{H} \alpha+[\mathrm{N}$ II $])$ line ratio in the northwest and southeast arms, in apparent contradiction to the observed $[\mathrm{O} \mathrm{III]} /(\mathrm{H} \alpha+[\mathrm{N} \mathrm{II}])$ distribution (Fig. 2). Either the northwest arm contains far more dust than the southeast arm, or there is foreground obscuration preferentially in front of the northwest side of the nucleus. We also cannot exclude intrinsically lower excitation conditions as the cause of the lower $[\mathrm{O} \mathrm{III}] /(\mathrm{H} \alpha+[\mathrm{N} \mathrm{II}])$ ratios and the absence of $[\mathrm{Ne} \mathrm{v}]$ emission in the northwest arms. The shorter wavelength emission lines we have observed originate from increasingly more highly ionized species; the effects of higher extinction and lower intrinsic ionization thus work in the same sense.

Beyond $\sim 5^{\prime \prime}$ radius, the line-emitting filaments are more luminous in [O III] than in $\mathrm{H} \alpha+[\mathrm{N} \mathrm{II]} \mathrm{both} \mathrm{north} \mathrm{and}$ south of the nucleus (Figs. 1 and 2). A-P96 presented longslit spectra and optical line ratios in the extended emission arcs. The narrow filament at the bottom of the emission-line images of Figure 1 (at $\Delta$ R.A., $\Delta$ decl. $\approx-3,-15.5$ ), labeled
"SH15" by A-P96, was analyzed in detail by those authors. We repeat some of their calculations of the physical conditions, because this feature appears narrow and filamentary in our HST images, rather than the intersection of a spherical shell and the ionization cone that they assumed. Focusing on the long, contiguous filament at the bottom of our images that appears to emit most of the $\mathrm{H} \alpha+[\mathrm{N}$ II] emission from the SH15 complex, the dimensions are $\sim 0$..3 $\times 8^{\prime \prime}$. Assuming the filament is cylindrical, the emitting volume is $V \sim 1.7 \times 10^{63} \mathrm{~cm}^{3} \approx 0.05 \mathrm{kpc}^{3}$. The $\mathrm{H} \alpha$ flux from this region is $F_{\mathrm{H} \alpha}=5.2 \times 10^{-15}$ ergs $\mathrm{cm}^{-2} \mathrm{~s}^{-1}$ (corrected for contamination by [N II] emission), corresponding to a luminosity of $L_{\mathrm{H} \alpha}=5.3 \times 10^{39} \mathrm{ergs} \mathrm{s}^{-1}$, and the [O III] $\lambda 5007$ flux is $F_{5007}=1.7 \times 10^{-14}$ ergs $\mathrm{cm}^{-2}$ $\mathrm{s}^{-1}$, corresponding to a luminosity of $L_{5007}=1.7 \times 10^{40}$ ergs $\mathrm{s}^{-1}$. Using equations (3) and (17), the electron densities derived from the $\mathrm{H} \alpha$ and $\lambda 5007$ luminosities are $3 /(f)^{1 / 2}$ $\mathrm{cm}^{-3}$ and $1.5 /(\eta f)^{1 / 2} \mathrm{~cm}^{-3}$, respectively. From equation (6), the mass of ionized gas in the SH15 filament is $M_{\text {ion }} \approx$ $5 \times 10^{6}(f)^{1 / 2} M_{\odot}$. From equation (7), we obtain $Q_{\text {rec }}=4.1$ $\times 10^{51}$ photons s ${ }^{-1}$ for SH15, leading to a value of $Q_{\text {ion }}=$ $5.2 \times 10^{54} \epsilon^{-1}$ photons $\mathrm{s}^{-1}$, assuming a cylindrical filament. Here $Q_{\text {ion }}$ is a lower limit, because we have used the projected distance of SH15 from the nucleus $(7 \mathrm{kpc})$, which will underestimate the true distance if the sight line from the nucleus to the SH15 filament is significantly out of the plane of the sky (see below). From equation (9), we find $\Gamma \approx 9 \times 10^{-3} \epsilon^{-1} f^{1 / 2}$ for the SH15 filament, roughly two times lower than for the southeast spiral arm, if the values of $\epsilon$ and $f$ are similar in the two regions. A drop in ionization parameter would be consistent with the decrease in the observed (i.e., uncorrected for reddening) $[\mathrm{O} \mathrm{III}] / \mathrm{H} \alpha$ ratio of $\sim 4.5$ (adjusted for [N II] emission) in the cloud in the southeast arm to $\sim 3.3$ in the SH15 filament. Our value of $Q_{\text {ion }}=9.4 \times 10^{53} \epsilon^{-1}$ photons $\mathrm{s}^{-1}$ calculated for the southeast circumnuclear spiral arm is a factor of $\sim 5$ lower than $Q_{\text {ion }}=5.2 \times 10^{54} \epsilon^{-1}$ photons $\mathrm{s}^{-1}$ found for the SH15 filament (assuming similar values of $\epsilon$ ). This may indicate that the circumnuclear spiral disk intersects the ionization cone near its edge and does not "see" the nuclear ionizing source as directly as some of the outer filaments such as SH15.

\subsection{Kinematics of the Gas: Two Inclined Disks}

Two prominent patterns in the F-P data are (1) the antisymmetry of the velocity field of the ionized gas across the nucleus (see Figs. 3-5), and (2) azimuthal velocity gradients along several arcs, including the circumnuclear spiral arms within $5^{\prime \prime}$ radius (see Fig. 4; right-hand panel). Given that the velocity field shows blueshifted (with respect to systemic) and redshifted gas on both sides of the nucleus, we discuss two types of models to explain the kinematics:

1. The gas follows circular orbits in the gravitational potential of the galaxy, in which case more than one rotating gaseous disk must be present. A single disk, whatever its rotation and orientation, will show redshifted velocities on one side and blueshifted velocities on the other. A warped disk would show a more complex velocity field, but the warping would need to be extremely convoluted to project large blueshifted and redshifted velocities on both sides of the nucleus.

2. Noncircular motions are present. For example, a conical, outflowing nuclear wind with sufficiently large 
opening angle and in an appropriate orientation could project both blueshifted and redshifted velocities on each side of the nucleus.

If we confine our attention to ionized gas, our analysis of the velocity field is overly restricted, because we see only the fraction of the gaseous arcs that fall within the bicone. To gain a more complete understanding of the gas distribution and kinematics, we therefore include the $21 \mathrm{~cm} \mathrm{H}$ I velocity field observed by Prieto \& Freudling $(1993,1996)$. The 21 $\mathrm{cm} \mathrm{H}$ I measurements have low spatial resolution (11" FWHM), but both the distribution and kinematics of the neutral hydrogen appear to join onto the ionized arcs in a continuous fashion. These observations suggest that the ionized bicone delineates the illuminated sectors of a larger gaseous structure (TT89). The combined Fabry-Perot and $21 \mathrm{~cm} \mathrm{H}$ I data sets now provide us with sufficiently complete spatial and velocity coverage to investigate detailed models of the gas motions.

\subsubsection{Photometric Constraints}

In order to understand the observed gas motions, we began with planar, circular disk models of the type described in Cecil, Wilson, \& Tully (1992) and adopted the following procedure:

1. We fitted isophotes to both ground-based and HST continuum images (TMWC) to derive the inclination of the stellar disk. The ellipticity $(e=1-b / a)$ increases from 0.4 at 0.5 radius to 0.54 at $\sim 20^{\prime \prime}$, then declines to 0.4 at $\sim 1^{\prime}$ radius. Assuming we are viewing an inclined circular disk, these values indicate an inclination of the disk spin axis to the line of sight of $54^{\circ}-63^{\circ}$. In the following, we adopt $63^{\circ}$.

2. The isophotal fits show that the P.A. of the major axis of the stellar disk decreases monotonically from $\sim 16^{\circ}$ close to the nucleus $\left(<1^{\prime \prime}\right)$ to $\sim 8^{\circ}$ at $\sim 1^{\prime}$ radius. This twist follows a "lazy $S$ " morphology, suggestive of a slightly warped stellar disk.

3. The HST emission-line images reveal a gas disk with major axis P.A. $\sim 135^{\circ}$ and inclination $\sim 40^{\circ}$. A similar major axis and inclination are obtained from the obscuring filaments seen against the background stellar light to the northwest of the nucleus (TMWC).

In summary, there is photometric evidence for two disks in NGC 5252: the stellar disk, whose spin axis is inclined $\sim 63^{\circ}$ to the line of sight and with photometric major axis projected along P.A. $\sim 15^{\circ}$; and the circumnuclear gas disk, which is inclined $\sim 40^{\circ}$ and with major axis projected near P.A. $\sim 135^{\circ}$.

\subsubsection{Kinematic Constraints and Disk Models}

We used the stellar velocities measured by HCC to obtain the rotation curve of the stellar disk of NGC 5252, assuming an inclination of $63^{\circ}(\S 4.2 .1)$. These absorptionline measurements of HCC span only the inner $20^{\prime \prime}-$ $30^{\prime \prime}$ radius of the galaxy, where starlight is sufficiently bright. To span the rest of the disk, we extrapolated beyond 30" with a flat rotation curve of semiamplitude $120 \mathrm{~km} \mathrm{~s}^{-1}$. The precise form of the extrapolation is not critical to the success of the model described below; qualitatively similar results are obtained with a falling rotation curve. We assume circular orbits and that the gravitational potential of the galaxy is spherically symmetric, so that the rotation curve is the same in different planes.
In this scenario, we found that we can attribute all observed gas motions (Fig. 4) to three systems in two disks:

1. Two high-velocity clumps-one $5^{\prime \prime}-10^{\prime \prime}$ north and blueshifted and the other $10^{\prime \prime}-13^{\prime \prime}$ south and redshifted (Figs 3 and 4)-suggest gas in a ring or torus residing in a plane close to the stellar disk and following the stellar rotation curve but counterrotating with respect to the stars. Our model of this component, extrapolated to the full extent of the stellar disk for clarity, is shown in Figure $6 a$ along with the observed velocity field. Such a counterrotating gas disk was suggested by HCC and A-P96 and has been observed in other S0 galaxies (e.g., Galletta 1987; Bertola et al. 1992).

2. Much of the gas on intermediate scales (radii $<40^{\prime \prime}$ ) appears to lie in a disk approximately coplanar with the small scale $\left(\sim 7^{\prime \prime}\right.$ diameter) spiral gas disk revealed by the $H S T$ observations, i.e., major axis P.A. $\sim 90^{\circ}-135^{\circ}$ and inclination $\sim 40^{\circ}$. The properties of this disk are further discussed below.

3. The outermost filaments at radii $>40^{\prime \prime}$ appear to rotate roughly in the plane of (and in the same sense as) the stellar disk. On this large scale, we therefore add a component representing gas coplanar and corotating with the stars.

The model velocity field obtained by superposing these three components is shown in Figures $6 c$ and $6 d$. This velocity field may be compared with the velocities observed in $\mathrm{H}$ I $21 \mathrm{~cm}$ emission (labeled contours in Fig. $6 c$ ) and in the ionized gas (Fig. 6a). It is evident that the combination of these three systems faithfully reproduces the observed motions, unlike the case of simple rotation (either with the stars or in the opposite sense; Fig. $6 a$ ), or some combination of outflow and rotation (Fig. $6 b$, discussed below), in the plane of the stellar disk. In fact, we find no compelling kinematic evidence for large-scale radial outflow from the nucleus.

Figure $6 b$ shows the velocity field expected for a model including radial outflow. The gas is confined to a single disk and undergoes both rotation and outflow in the disk plane. The parameters are chosen to reproduce the observed velocities at large radii. However, the observed relatively small azimuthal velocity gradients along many of the arcs within the bicone, the larger radial gradients down the axis of the cone, and a broad strip of near-systemic gas motions almost north-south are completely at odds with this model. Large radial motions are not expected in the neutral gas, so models invoking such motions for the ionized gas have difficulty accounting for the close kinematic association between the neutral and ionized gases.

As discussed above, we require that the second gaseous disk be inclined by $\sim 40^{\circ}$ and have a major axis along P.A. $\sim 135^{\circ}$ at small radii (within a few arcseconds of the nucleus). This orientation is consistent with that inferred for the circumnuclear spiral gas disk observed with $H S T$. In our preferred model (Figs. $6 c$ and $6 d$ ), we have smoothly decreased the major axis P.A. of this gaseous disk to P.A. $\sim 90^{\circ}$ beyond $\sim 5^{\prime \prime}$ radius in order to account for the velocity field of most of the gas in the bicone. Figure $6 c$ shows that the model is consistent with the limited information available on the $\mathrm{H}$ I velocity field. Further models (not presented here) showed that alternative inclination angles or additional skewing of the disk major axis P.A. by more than $\pm 4^{\circ}$ are excluded because they project the motions close to systemic velocity in the wrong place or deviate from 


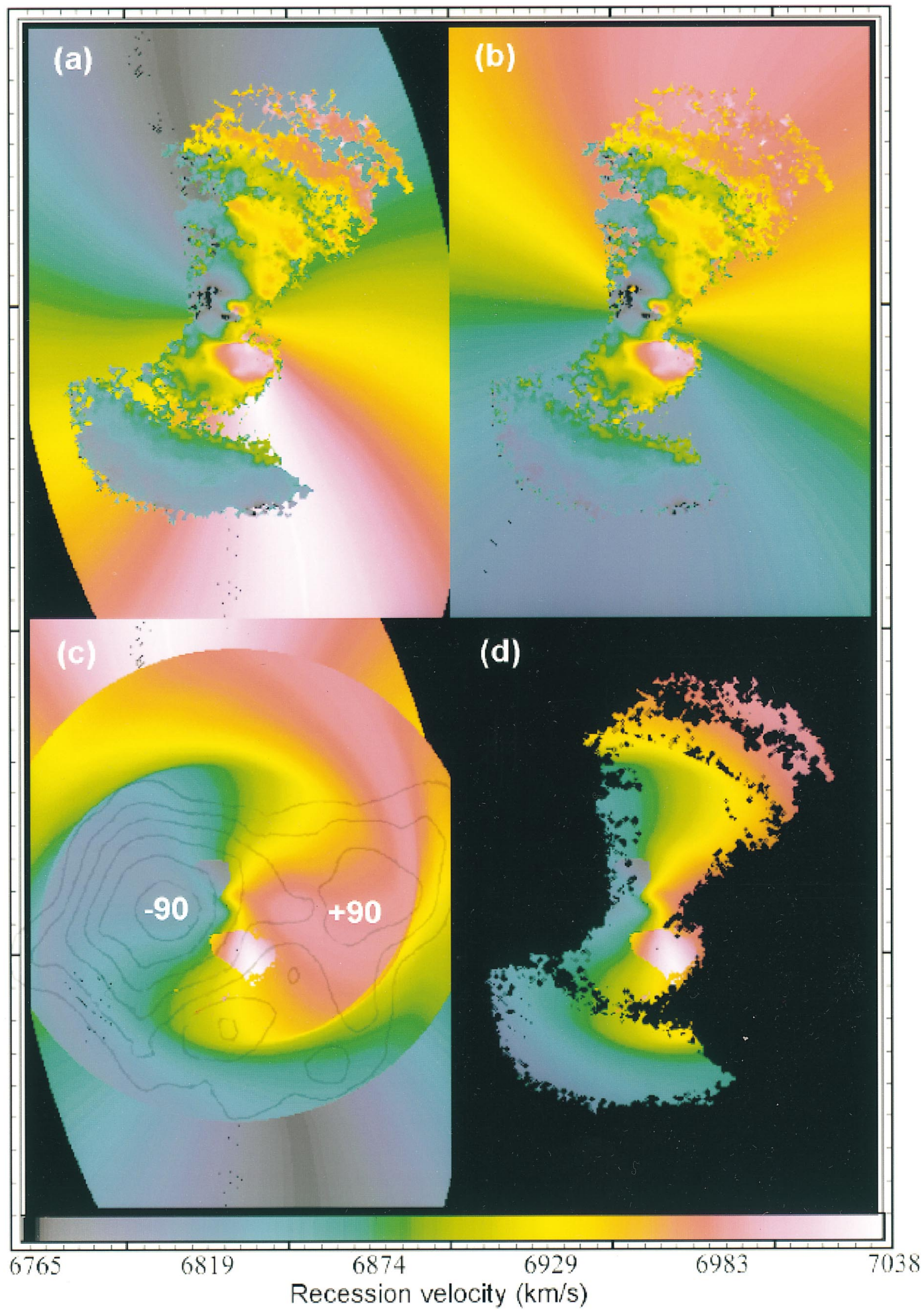

Fig. 6.-Predicted radial velocities (see the color bar at the bottom) for various kinematic models of gas motions in NGC 5252. The observed velocities from the [O III] line emission are superposed in $(a)$ and $(b)$. Flux contours of H I $21 \mathrm{~cm}$ emission(Prieto \& Freudling 1996) are shown in (c). Rectangular field of view is $92.6 \times 131$ ".7 (R.A. $\times$ decl.). (a) Gas in the stellar disk plane following the stellar rotation curve, but with the gas counterrotating with respect to the stars. The inclination was derived from photometry of optical continuum images (see text). The model describes well the observed gas motions $\sim 10^{\prime \prime}-15^{\prime \prime}$ from the nucleus. The slight deviation of the kinematic major axis from a straight line results from the warping inferred from the isophote fitting (see $\S 4.2 .1$ ). Most of the ionized gas motions deviate from this model. (b) Rotating gas with an additional $80 \mathrm{~km} \mathrm{~s}^{-1}$ gaseous outflow within the disk plane. This disk is oriented with the same major axis as the stellar disk and rotates in the same sense as the stars, but has rotation axis inclined $35^{\circ}$ from our line of sight. With these parameters, the maximum radial velocities project close to the ionization cone axis and are forced to agree with those observed at large radii in the ionized gas. However, the model accounts for neither the $\mathrm{H}$ I kinematics nor the ionized gas kinematics through most of the bicone. (c) Inside the circular area, velocity field of a model combining (1) patches of counterrotating gas in the stellar disk plane (from the model in [a]), (2) gas in a disk with major axis P.A. $90^{\circ}-135^{\circ}$ and inclination $40^{\circ}$, and (3) at large radii, gas in the plane of the stellar disk and rotating with the stars. $\mathrm{H}$ i flux contours and labeled peak velocities (in $\mathrm{km} \mathrm{s}^{-1}$, relative to the galaxy systemic velocity) are superposed. The velocity field outside the circular area is the model describing the rotation of the stars. (d) Model velocity field for the ionized gas from the composite model shown in $(c)$. This model velocity field may be compared with the observed velocity field shown in $(a)$. 
the measured $\mathrm{H}$ I velocities. In our model, the disk with P.A. $\sim 90^{\circ}$ is truncated at radii $\simeq 40^{\prime \prime}$; as noted above, gas at still larger radii appears to rotate roughly in the plane of (and in the same sense as) the stellar disk. This truncation accounts for the rapid swing in the model's major axis P.A. from $\sim 90^{\circ}$ to $\sim 195^{\circ}$ between radii of $40^{\prime \prime}$ and $50^{\prime \prime}$ (Figs $6 c$ and $6 d$ ). It is unclear whether the two gas disks are physically separate and simply superposed along the line of sight or there is actually a large warp between $40^{\prime \prime}$ and $50^{\prime \prime}$ radii. Despite this uncertainty, the presence of two highly inclined gas disks must project gas in many different directions as seen from the nucleus, allowing the bicone of ionizing radiation to be clearly traced by the ionized gas. The spectacular nature of the extended nebulosity in NGC 5252 may thus result from the presence of the second disk.

Based on the model shown in Figures $6 c-6 d$, we can draw a kinematical connection between the circumnuclear spiral gas disk, most of the gaseous arcs within the bicone, and the neutral hydrogen gas in the western and eastern quadrants. The blueshifted $\mathrm{H}_{\mathrm{I}}$ emission observed $\sim 50^{\prime \prime}$ south of the nucleus can be associated with the southernmost ionized gas, which appears to rotate in the plane of the stellar disk. As mentioned above, while the kinematics of the large-scale gas may be well described by two separate disks superposed along the line of sight, it is also possible that the extended gas represents a continuous, highly warped disk. For example, observations of the large-scale $\mathrm{H}$ i gas distribution in the S0 galaxy NGC 4753 (Steiman-Cameron, Kormandy, \& Durisen 1992) show that differential precession may produce significant contortions and abrupt twists in a gaseous disk.

\subsubsection{Gas Settling}

A substantial fraction of elliptical and S0 galaxies exhibit stellar "shells," starburst rings, gaseous rings, and $\times$ shaped structures (e.g., Schweizer \& Seitzer 1988; Whitmore \& Bell 1988). Van Driel \& van Woerden (1991) summarize the distribution and kinematics of $\mathrm{H}$ I gas in a sample of 24 early-type ( $\mathrm{S} 0$ to $\mathrm{Sa}$ ) galaxies. These galaxies often contain inner and outer $\mathrm{H}$ I rings. Most of the inner rings appear to be contained within or are tilted slightly from the stellar disk. Nearly $50 \%$ of the outer rings are tilted $25^{\circ}$ to $55^{\circ}$ out of the plane of the stellar disk, suggesting warped $\mathrm{H}$ I distributions (e.g., NGC 4230; van Driel et al. 1988). The H I kinematics are well described by circular rotation with essentially flat rotation curves that imply massive dark haloes. The $\mathrm{H}$ I column densities are typically too low to sustain large-scale star formation. Van Driel \& van Woerden (1991) consider the most likely origins of the H I gas in S0 galaxies to be either (1) gas accreted from galaxygalaxy encounters (e.g., see Mihos \& Hernquist 1996 and references therein), or (2) leftover gaseous rings that have not been consumed by star formation. A feature of models of galaxy-galaxy encounters and mergers (e.g., Mihos, Walker, \& Hernquist 1995; Thakar \& Ryden 1996; Barnes \& Hernquist 1996) is that the gas settles into an inclined plane, possibly counterrotating, depending on whether the encounter was prograde or retrograde. Gravitational torques can cause precession of the spin axis of the gas disk.

The $\mathrm{H} \mathrm{I}$ and ionized gas distributions and kinematics in NGC 5252 are consistent with the observations of other gas-rich S0s mentioned above. Given the prevalence of warped or counterrotating gaseous ring structures in recent simulations of galaxy-galaxy encounters, we favor the accretion hypothesis as an explanation for the structure of
NGC 5252. The observed debris from the putative galaxygalaxy encounter is entirely gaseous, and some of it happens to be illuminated by the AGN. Most of the gas appears to lie outside the ionization bicone and is probably neutral. Further improvements in the $\mathrm{H}_{\mathrm{I}} 21 \mathrm{~cm}$ spatial resolution and sensitivity will better probe the merger history and the overall gravitational potential of the galaxy. The absence of close companion galaxies to NGC 5252 (Freudling \& Prieto 1996) and the apparently well-ordered velocity field of the gas suggest that the merger or encounter occurred in the not too recent past.

\section{CONCLUSIONS}

We have presented HST WFPC2 images and the twodimensional velocity field of the ionized gas in the type 2 Seyfert galaxy NGC 5252. The HST images, including a new [O III] $\lambda 5007$ emission-line image obtained through a narrowband filter, show the extended arcs to consist of a complex network of filamentary strands. Comparison with the two-dimensional velocity field shows that adjacent filaments often have very different kinematics.

The circumnuclear spiral gas disk, previously discussed by Tsvetanov et al. (1996), shows a progressive decrease in the $\left[\begin{array}{ll}\mathrm{O} & \mathrm{III}\end{array}\right] \lambda 5007 /\left(\mathrm{H} \alpha+\left[\begin{array}{ll}\mathrm{N} & \mathrm{II}\end{array}\right]\right)$ line ratios across the nucleus from southeast to northwest; this decrease may result from a gradient of extinction either within the disk or foreground to it. Alternatively, the $[\mathrm{O} \mathrm{III}] /(\mathrm{H} \alpha+[\mathrm{N} \mathrm{II}])$ line ratios may be intrinsically lower in the northwest arm if this material has a lower ionization parameter, perhaps because it lies closer to the edge of the ionization cone than the southeast arm. The electron densities in the circumnuclear spiral arms derived from the $\mathrm{H} \alpha$ and [O III] $\lambda 5007$ line fluxes are a few $\mathrm{cm}^{-3}$.

In contrast to previous attempts to interpret the motions of the arcuate gas filaments within the bicone as radial flows, our more complete kinematic data, in conjunction with the H I data of Prieto \& Freudling (1993, 1996), show that the gaseous kinematics may be fully explained in terms of circular motions in two disks, each following the same rotation curve as the stars. One disk coincides with the stellar disk and contains gas that counterrotates at $\sim 10^{\prime \prime}-$ $15^{\prime \prime}(4.5-6.7 \mathrm{kpc})$ radius and corotates with the stars at large radii $\left(>40^{\prime \prime}=18 \mathrm{kpc}\right)$. The second disk is inclined at a large angle to the stellar disk, and its plane is broadly consistent with that defined by the small-scale $\left(7^{\prime \prime}=3.1 \mathrm{kpc}\right)$ spiral gas disk observed in HST images. This second disk includes the circumnuclear spiral arms, most of the extended ionized filaments, and the $\mathrm{H}$ I gas observed in $21 \mathrm{~cm}$ line emission outside the ionization bicone. The presence of a gas disk at a large angle to that of the stellar disk strongly suggests a recent merger, perhaps with a gas-rich dwarf galaxy. Dissipative processes may then provide the infalling gas necessary to fuel the nuclear activity. The absence of significant radial motions, together with the well-defined ionization cones, suggests that the gas is photoionized by a compact nuclear source rather than being ionized in situ by shock waves.

We thank the technical staff at CTIO and Ted Williams (Rutgers) for their assistance with the F-P observations, and also the referee, R. Pogge (Ohio State), for helpful discussions and a thorough reading of the manuscript. This work has been supported by grants NAGW-2689 and NAGW-3268 from NASA, and by grants GO-05426-93A and GO-05846-94A from STScI. 


\section{REFERENCES}

Acosta-Pulido, J. A., Vila-Vilaró, B., Pérez-Fournon, I., Wilson, A. S., \& Tsvetanov, Z. I. 1996, ApJ, 464, 177 (A-P96)

Allen, C. W. 1973, Astrophysical Quantities (London: Athlone)

Atherton, P. D., Taylor, K., Pike, C. D., Harmer, C. F. W., Parker, N. M., \& Hook, R. N. 1982, MNRAS, 201, 661

Barnes, J. E., \& Hernquist, L. 1996, ApJ, 471, 115

Bertola, F., Buson, L. M., \& Zeilinger, W. W. 1992, ApJ, 401, L79

Binette, L., Wilson, A. S., \& Storchi-Bergmann, T. 1996, A\&A, 312, 365

Biretta, J., et al. 1996, WFPC2 Instrument Handbook, Version 4.0 (Baltimore: STScI)

Bland, J., \& Tully, R. B. 1989, AJ, 98, 723

Cappi, M., Mihara, T., Matsuoka, M., Brinkman, W., Prieto, M. A., \& Palumbo, G. G. C. 1996, ApJ, 456, 141

Cecil, G., Bland, J., \& Tully, R. B. 1990, ApJ, 355, 70

Cecil, G., Wilson, A. S., \& Tully, R. B. 1992, ApJ, 390, 365

Dopita, M. A., \& Sutherland, R. S. 1995, ApJ, 455, 468

Freudling, W., \& Prieto, M. A. 1996, A\&A, 306, 39

Galletta, G. 1987, ApJ, 318, 531

Hartigan, P., Morse, J. A., Heathcote, S., \& Cecil, G. 1993, ApJ, 414, L121

Heathcote, S., Morse, J. A., Hartigan, P., Reipurth, R., Schwartz, R. D., Bally, J., \& Stone, J. 1996, AJ, 112, 1141

Held, E. V., Capaccioli, M., \& Cappellaro, E. 1992, in Testing the AGN Paradigm, ed. S. Holt, S. Neff, \& C. M. Urry (New York: AIP), 613 (HCC)

Ho, L. C., Filippenko, A. V., \& Sargent, W. L. W. 1993, ApJ, 417, 63

Holtzman, J. A., et al. 1995, PASP, 107, 156

Huchra, J., \& Burg, R. 1992, ApJ, 393, 90

Mendoza, C. 1983, in IAU Symp. 103, Planetary Nebulae, ed. D. R. Flower (Dordrecht: Reidel), 143
Mihos, J. C., \& Hernquist, L. 1996, ApJ, 464, 641

Mihos, J. C., Walker, I. R., \& Hernquist, L. 1995, ApJ, 447, L87

Morse, J. A., Hartigan, P., Cecil, G., Heathcote, S., \& Raymond, J. C. 1992, ApJ, 399, 231

Morse, J. A., Heathcote, S., Cecil, G., Hartigan, P., \& Raymond, J. C. 1993, ApJ, 410, 764

Morse, J. A., Raymond, J. C., \& Wilson, A. S. 1996, PASP, 108, 426

Morse, J. A., Winkler, P. F., \& Kirshner, R. P. 1995, AJ, 109, 2104

Osterbrock, D. E. 1989, Astrophysics of Gaseous Nebulae and Active Galactic Nuclei (Mill Valley: Univ. Sci. Books)

Prieto, M. A., \& Freudling, W. 1993, ApJ, 418, 668

. 1996, MNRAS, 279, 63

Schweizer, F., \& Seitzer, P. 1988, ApJ, 328, 88

Steiman-Cameron, T. Y., Kormendy, J., \& Durisen, R. H. 1992, AJ, 104, 1339

Tadhunter, C. N., Robinson, A., \& Morganti, R. 1989, in Extranuclear Activity in Galaxies, ed. E. J. A. Meurs \& R. A. E. Fosbury (Garching: ESO), 293

Tadhunter, C., \& Tsvetanov, Z. 1989, Nature, 341, 422 (TT89)

Thakar, A. R., \& Ryden, B. S. 1996, ApJ, 461, 55

Tsvetanov, Z. I., Morse, J. A., Wilson, A. S., \& Cecil, G. 1996, ApJ, 458, 172 (TMWC)

Tsvetanov, Z. I., et al. 1998, in preparation

van Driel, W., \& van Woerden, H. 1991, A\&A, 243, 71

van Driel, W., van Woerden, H., Gallagher, J. S., III, \& Schwarz, U. J. 1988, A\&A, 191, 201

Whitmore, B. C., \& Bell, M. 1988, ApJ, 324, 741

Wilson, A. S., \& Tsvetanov, Z. I. 1994, AJ, 107, 1227 (WT94) 